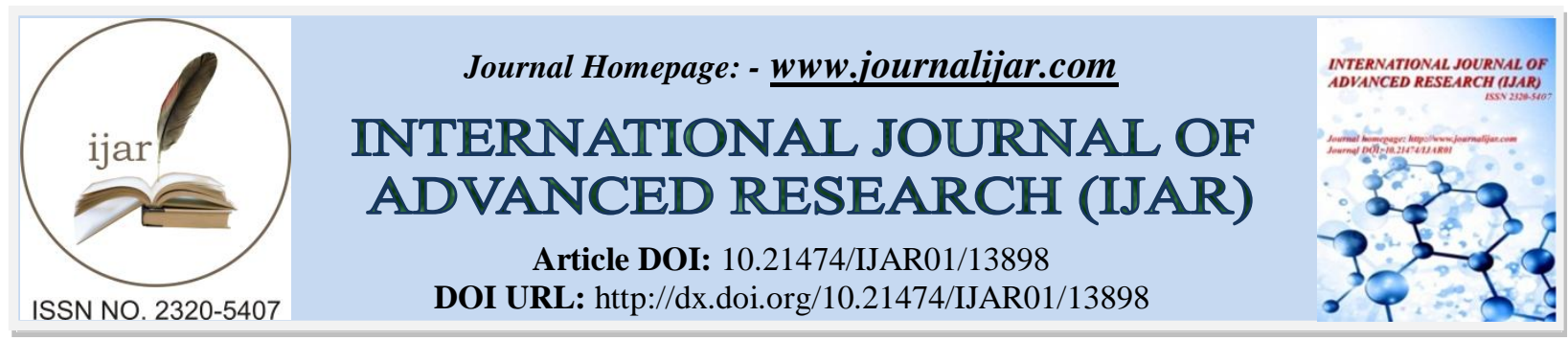

RESEARCH ARTICLE

\title{
PANCHTATAVAS - MYSTERIOUS WORLD OF MATTER
}

V.P. Srivastava and Rahul Singh

Department of Physics, St. Andrew's College, Gorakhpur 273001, India.

\section{Manuscript Info}

\section{Manuscript History}

Received: 05 October 2021

Final Accepted: 10 November 2021

Published: December 2021

Key words:-

Panchtatavas, Parmanu, Atoms, Quarks, Gluon

\begin{abstract}
According to Ancient Indian philospers all matter of this universe is made up of five basic constituents commonly called 'panch tatavas'. Indian philospher Maharishi Kanad around 500 BC postulated that matter is divisible. The smallest indivisible matter were named as 'parmanu'. Greek philosphers named this indivisible particles 'atoms'. In 1808 John Dalton suggest that the atom was indivisible and industructible. After the discovery of electron and proton inside the atom, led to the failure of Dalton's theory. In the thirty years between the discovery of neutron in 1932 to the middle of 1962, more than thirty new particles were found, which were thought to be the builders of matter in the universe. Therefore it was established beyond doubt that all the particles discovered are not fundamental but have complex nature. In 1964 Gell-Mann and Zweing proposed that most of these particles called 'Quarks'. Nearly 200 elementary particles were discovered so far. It appears that our search for the ultimate builder is not in a concluding stage but we are reaching towards the goal in stages. In this way we can say that the mysterious world of elementary particles and Quark is still mysterious. The same is written in 'Vedas' and 'Purana' that the search of ultimate particle will go on and on.
\end{abstract}

Copy Right, IJAR, 2021,. All rights reserved.

\section{Introduction:-}

When we look at our surroundings, we see a large number of things of different sizes, shapes, apperance and texture. Everthing in the universe is made up of material which is called matter. The food we eat, the clothes we wear, the air we breathe, the houses where we live in, plants, animals, stars etc, each thing is made up of matter. So we can say everything in this universe is made of matter. Matter may be defined as anything that occupies space, possess mass and the presence of which can be felt by any or more of our five senes. (i.e. sight, touch, smell, hearing and taste). It is important to note that vaccum is not a matter since it neither occupies space nor has mass. Similarly heat, light, sound, electricity, magnetism etc are not considered as matter because they are massless and do not occupy space.

According to ancient Indian philosophers all matter whether living or non-living is made up of five basic coustituents commonly called 'panchtatavas' i.e. air, earth, fire, sky and water [1]. Ancient Indian and Greek philosophers have been thinking about what matter is ultimately made up of. It was around $500 \mathrm{BC}$ that an Indian philospher, Maharshi kanad had postulated that matter is divisible i.e. if we go on breaking matter, we will get smaller and smaller particles and ultimately, the particles obtained may be so small that they cannot be further divided. These indivisible paticles were named as "parmanu" [2-3]. Greek philosphers Democritus and Leucippus, during the same period gave the same idea. They called the smallest indivisible particles as "atoms" (Greek means 
un-cutable)[4]. It was also suggested by Indian philospher, Pakudha Katyama. during the same priod, that in some cases, the atoms may not exist in the free state but may exist in the combined state in the form of species called molecules or compounds [5]. However all these were simply guess upto the eighteenth century, there were no experimental methods and results avilable to support the above philosophical ideas.

British Chemist John Dalton gave the basic theory about the nature of matter in 1808. Dalton take the idea of divisiblility of matter, which was philosophy till then. He said that the smallest particles of matter are 'atoms'. According to Dalton's atomic theory all matter, whether an element, a compound or a mixture is composed of small particles called atoms [6-7]. This theory suggested that the atom was indivisible and indestructible. But the discovery of two fundamental particles electrons and protons inside the atom, led to the failure of this aspect of Dalton's atomic theory.

In 1897 when electron was discovered by Thompson existence of photon was realised by Planck in 1901 and confirmed by Einsteen in 1905 [8-10]. The existance of proton within the nucleus was established by Rutherford and his co-workers in 1912 [11]. Rutherford discussed the existence of neutral particle called neutron and ultimately its existence was confirmed by Chadwick in 1932 [12]. Most of the particles discovered later were found in cosmic rays and were identified through their tracks in photographic emulsion plate or closed chamber. Around 1932, Anderson in USA and Blackett in England discovered independently a particle named positron which has same rest mass as electron but charge equal and opposite to it. Yukawa in 1935 predicted the existence of meson for mediating the force between nucleous [13].

In the thirty years between the discovery of neutron in 1932 to the middle of 1962, more than thirty new particles were found, which were thought to be the builders of matter in the universe. The end is still not in sight such large number of particles can not be ultimate builders or fundamentals and there arose serious doubt about them. Ultimately, in late sixties it was established beyond doubt that all the particles discovered are not fundamental but have complex structure. Murray Gell - Mann and G - Zweig in 1964 proposed that most of these particles are made up of more fundamental particles called 'Quarks' [14-15]. They are bound by an exchange of a particle called 'gluon'. Existence of gluon has been confirmed in 1979 by combined efforts of Scientist from all over the world in Germany [16]. In the simplest version, there are three species of quarks and corresponding antiquarks. Quarks and antiquarks have opposite parity. The three quarks initially proposed were named up, down and strange. These quarks were sufficient for describing all particles known till the end of 1974. For example a proton is composed of two up quarks, one down quark as shown in figure 1. A gluon is an elementary particle that acts as the exchange particle for strong force between quarks Gluons binds quarks together to form protons and neutrons as shown in fig. 2 . It shows annihilation of electron and positron emitted gluons are represented as helices. However, some new elementary particles were discovered afterwards and existence of two more quarks have been proposed. These two quarks are charm and Beauty. A sixth one named Top first observed at Fermilab in 1995, was the last to be discovered [17]. Nearly 200 elementary particles discovered so far [18-19].

In this way we may conclude that the mysterious world of elementary particles and Qurak is still mysterious. It appears that our search for the ultimate builder is not in a concluding stage but we are reaching towards the goal in stages. How far one has to go is the mystery of almighty nature. The same is written in Vedas and Purana that the search of Ultimate builder will go on and on.

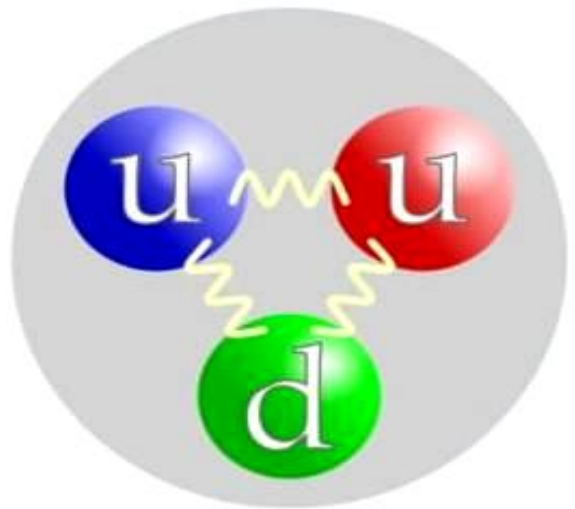

Fig. 1:- Quark Model for Proton. 


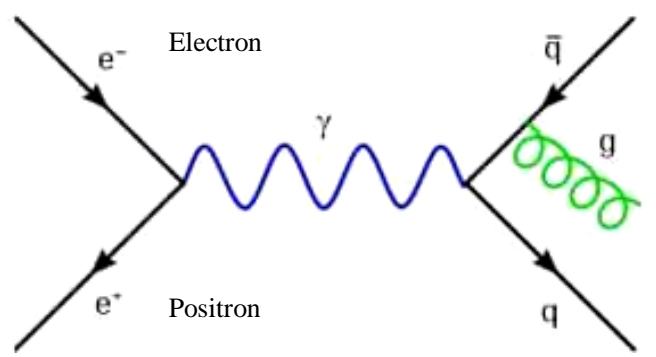

Fig. 2:- Annihilation of an electron and positron with emission of gluon (g).

\section{Refrences:-}

(1). Rosen, Steven J (1994) Sri Pancha Tattva : The Five Features of God Folk Books, New York. ISBN : 09619763-7-3

(2). KaK, S. 'Matter and Mind : The Vaishashika Sutra of Kanada' (2 - 16), Mount Menu Publishing Mississuga, Ontario ISBN : 978-1- 988207-13-1.

(3). James G. Lochtefeld (2002) The Illustrated Encyclopedia of Hindusim : N-Z the Rosen Publishing pp 729 ISBN : 978-08239-3180-4.

(4). Alexandra A. Radzig, Boris M. Smirnov, Springer Series in Chemical Physics 31, ISBN : 978-3-642-82048-9

(5). Lokayata A (1959) Study In Ancient Indian Materialism People Publishing House, p. 517. ISBN : 9788170070061

(6). A Dictionary of Physics (6ed) (2004) Oxford Univesity Press, ISBN - 13 : $978019923399-1$

(7). Kenny, Anthoney (2004). Ancient Philosophy A New History of Western Philosophy 1. Oxford, England : Oxford University Press. pp26-28, ISBN 0-19-875273-2

(8). David L. Anderson (1964). The Discovery of the Electron Princeton, N.J : Van Nostrand.

(9). Isaacson, Walter (2007). Einstein : His life and Universe Simon \& Schuster. ISBN : 978-0-7432-6473-0

(10). Griffiths, David J. (2008). Introduction to Elementary Particles (2nd revised ed.). WILEY-VCH. ISBN 978-3-52740601-2

(11). Romer A (1997). "Proton or prouton ? Rutherford and the depths of the atom." American Journal of Physics. $65(8): 707$

(12).Chadwick, James (1932) : "Existence of a Neutron" Proceedings of the Royal Socienty A. 136 (830) : 692-708.

(13). Sylvie Braibant, Giorgio Giacomelli, Maurizio Spurio (2012). Particles and Fundamental Interactions : An Introduction to Particle Physics (Ist ed). Springer. p-1 ISBN : 978-94-007-2463-1

(14). Gell-Mann, M. (1964) Physics Letters 8 (3) : 214-215

(15). Zweing, G. (1964) : CERN Report No. 8419/TH.412

(16). Soding P. (2010) : European Physical Journal H, 35(1) : 3-28.

(17). Anderson C.W., et al (2018) Phys. Rev D97. 1, 014506.

(18). Abachi, S. et al (1995). Physical Review Letters 74 (14) 2632-2637

(19). Zyla P.A; et al (2020), "Review of Particle Physics". Progress of theoretical and Experimental physics. $083 \mathrm{C} 01$. 